\title{
Construindo o conhecimento em saúde coletiva: uma análise das teses e dissertações produzidas
}

\author{
BUILDING KNOWLEDGE IN COLLECTIVE HEALTH: AN ANALYSIS OF THESIS AND DISSERTATIONS \\ CONSTRUYENDO EL CONOCIMIENTO EN SALUD COLECTIVA: \\ UNAANÁLISIS DE LAS TESIS DEL DOCTORADO Y MAESTRÍA PRODUCIDAS
}

\section{Emiko Yoshikawa Egry'1 ${ }^{1}$ Rosa Maria Godoy Serpa da Fonseca², Maria Rita Bertolozzi ${ }^{3}$, Maria Amélia de Campos Oliveira ${ }^{4}$, Renata Ferreira Takahashi ${ }^{5}$}

\author{
1 Enfermeira. Professora \\ Titular do Departamento de \\ Enfermagem em Saúde \\ Coletiva da Escola de \\ Enfermagem da Universi- \\ dade de São Paulo (EEUSP). \\ Coordenadora da Área \\ de Concentração em \\ Saúde Coletiva do Programa \\ de Pós-Graduação em \\ Enfermagem. \\ emiyegry@usp.br \\ 2 Enfermeira. Professora \\ Titular do Departamento \\ de Enfermagem em Saúde \\ Coletiva da EEUSP. \\ Vice-diretora da EEUSP. \\ rmgsfon@usp.br \\ 3 Enfermeira. Professora \\ Doutora do Departamento \\ de Enfermagem em Saúde \\ Coletiva da EEUSP. \\ Vice-coordenadora da Área \\ de Concentração em Saúde \\ Coletiva do Programa de \\ Pós-Graduação em \\ Enfermagem. \\ mrbertol@usp.br \\ 4 Enfermeira. Professora \\ Doutora do Departamento \\ de Enfermagem em Saúde \\ Coletiva da EEUSP. \\ Presidente da Comissão de \\ Graduação da EEUSP. \\ macampos@usp.br \\ 5 Enfermeira. Professora \\ Doutora e Chefe do \\ Departamento de \\ Enfermagem em Saúde \\ Coletiva da EEUSP. \\ rftakaha@usp.br
}

\section{RESUMO}

Realizou-se um estudo com objetivo de analisar a produção do saber da enfermagem em saúde coletiva, resultante da pósgraduação vinculada ao Departamento de Enfermagem em Saúde Coletiva da Escola de Enfermagem da Universidade de São Paulo, no período de 1992 a 2004 . Foram produzidas 86 teses e dissertações sobre múltiplos objetos, principalmente sobre os perfis epidemiológicos e de reprodução social, articulados aos processos de saúde-doença dos grupos sociais. Houve predomínio de análises qualitativas e do referencial teórico-metodológico materialista-histórico e dialético. Grupos populacionais de determinados territórios constituíram a fonte empírica mais freqüente. Concluiu-se que a adoção de categorias analíticas relativas à produção e reprodução social, assim como gênero e geração, possibilitaram a aproximação real do objeto para interpretar e intervir, contribuindo para o avanço dos conhecimentos em saúde coletiva.

\section{DESCRITORES}

Enfermagem em saúde pública. Educação de pós-graduação em enfermagem.

Pesquisa em enfermagem.

\section{ABSTRACT}

The aim of this study was to analyze scientific production in Collective Health developed by Collective Health Nursing Department of the School of Nursing Sao Paulo University within 1992 and 2004. A total of 86 master dissertations and doctoral thesis were produced, concerning a variety of study objects, mainly epidemiologic profiles and social reproduction, connected to health-illness processes of social groups. Qualitative analysis predominated, as well as the historical and dialectical theoretical referential. Empirical data were obtained predominantly from different social groups of specific territories. The adoption of analytical categories such as social production and reproduction, gender and generation enabled approaching to comprehend and propose intervention on the study objects, thus improving Collective Health scientific knowledge.

\section{RESUMEN}

En el presente estudio se tuvo como objetivo analizar la producción del saber de la enfermería en salud colectiva, resultante del post grado vinculado al Departamento de Enfermería en Salud Colectiva de la Escuela de Enfermería de la Universidad de São Paulo, en el período de 1992 al 2004. Fueron producidas 86 tesis de maestría y doctorado sobre múltiples objetos, principalmente sobre los perfiles epidemiológicos y de reproducción social, articulados a los procesos de salud-enfermedad de los grupos sociales. Hubo predo-minio de análisis cualitativos y del referencial teórico-meto-dológico, materialista-histórico y dialéctico. Grupos poblacionales de determinados territorios constituyeron la fuente empírica más frecuente. Se concluyó que la adopción de categorías analí-ticas relativas a la producción y reproducción social, así como género y generación, hicieron posible la aproximación real del objeto para interpretar e inter-venir, contribuyendo en el avance de los conocimientos en salud colectiva.

\section{KEY WORDS}

Public health nursing. Education, nursing, graduate. Nursing research.

\section{DESCRIPTORES}

Enfermería en salud pública. Educación de post-grado en enfermería. Investigación en enfermería. 


\section{INTRODUÇÃO}

Em fevereiro de 1987, a Escola de Enfermagem da Universidade de São Paulo constituiu o seu quarto e último departamento, o de Enfermagem em Saúde Coletiva - ENS ${ }^{(a)}$, reunindo três núcleos disciplinares originários do Departamento de Enfermagem Médico-Cirúrgica, a saber: Nutrição, Doenças Transmissíveis e Enfermagem Preventiva e Comunitária ${ }^{(1)}$.

O principal desafio foi a construção do saber, sob a égide do marco teórico-metodológico da Saúde Coletiva, na perspectiva do materialismo-histórico e dialético. O cenário político da época propugnava a reorientação do paradigma da saúde ao recolocar o campo da Saúde Coletiva como um lócus potente e fecundo, onde se debatia a necessidade de transformação da situação social e econômica da década de 70, do século passado, principalmente no âmbito da América Latina.

A mudança paradigmática do campo da saúde demandava a qualificação das docentes para a renovação dos conhecimentos, assim como para responder às demandas nacionais que, por força do movimento da Reforma Sanitária, haviam se traduzido na nova Constituição brasileira, o que possibilitou, em 1990, a criação do Sistema Único de Saúde ${ }^{(2)}$.

O Programa de Pós-graduação da Escola de Enfermagem havia sido instituído em 1973, com cinco áreas de concentração. A criação da área de Enfermagem em Saúde Coletiva, em 1991, representou a primeira modificação ao longo de quase duas déca$\mathrm{das}^{(3)}$. A primeira turma de mestrandas da área, com apenas três alunas regularmente matriculadas ${ }^{(b)}$, iniciou as atividades no dia 12 de março de 1992 e cumpriu um extenso calendário de disciplinas obrigatórias da própria área de concentração e do domínio conexo. À semelhança das demais áreas do Programa, uma carga horária expressiva era desenvolvida em disciplinas: 24 créditos $^{(\mathrm{c})}$ obrigatórios da área e 14 créditos obrigatórios do domínio conexo, nas disciplinas de Metodologia da Pesquisa em Enfermagem, com seis créditos, e Problemas do Ensino de Enfermagem, com oito.

As disciplinas obrigatórias da área de concentração eram: Enfermagem em Saúde Coletiva I e Enfermagem em Saúde Coletiva II, com 12 créditos cada. As disciplinas eletivas incluíam Temas Avançados em Saúde Coletiva, com oito créditos; Metodologia de Investigação em Saúde Coletiva, com oito créditos, e Tendências da Investigação em Saúde Coletiva, com um crédito.
As docentes responsáveis por planejar, organizar, ministrar e avaliar o curso, bem como pela orientação das discentes da área, eram egressas dos programas de doutorado de diferentes unidades da Universidade de São Paulo.

A Área organizou-se sob a vertente teórico-filosófica do materialismo-histórico e dialético (MHD) tendo como premissas ${ }^{(2)}$ :

- a expressão do processo saúde-doença em saúde coletiva, bem como as políticas sociais e de saúde, são determinados socialmente;

- as práticas em saúde coletiva são práticas sociais, construídas em diferentes processos de trabalho, estreitamente articuladas à estrutura da sociedade e à dinâmica das forças de seus grupos sociais;

- os processos de trabalho no âmbito do ensino, da assistência e da investigação científica na saúde e na Enfermagem comportam transformações historicamente determinadas e de caráter processual;

- a construção do saber em saúde coletiva é um processo coletivo, que tem como horizonte a transformação da realidade.

Doze anos após o início das atividades da área, muitas mudanças foram implementadas em decorrência das avaliações, tanto internas ao Departamento de Enfermagem em Saúde Coletiva, quanto as originárias dos organismos nacionais de fomento à pós-graduação. Outras mudanças ocorreram em virtude de modificações do perfil do público, que passou a demandar qualificação em nível de pós-graduação, constituído inicialmente por docentes de escolas públicas de terceiro grau em Enfermagem, em seguida por trabalhadores de serviços públicos de saúde e, finalmente, por docentes oriundas de instituições privadas de ensino e assistência.

Uma das mudanças mais notáveis foi decorrência da redução drástica da duração dos cursos de mestrado no País, de 4 para 2 anos, à qual o Departamento teve de se adequar, uma vez que constitui um dos critérios de avaliação de maior peso quando é julgada a agilidade dos programas. Como resultado, houve necessidade de redução do número de créditos exigidos para a conclusão do programa. Este enxugamento trouxe dificuldades, pois persistem lacunas de conhecimentos na formação dos profissionais da saúde acerca dos fundamentos da Saúde Coletiva, ainda que o Sistema Único de Saúde encontre-se mencionado na maioria dos currículos plenos de formação de terceiro grau das profissões da saúde.
Construindo o conhecimento em saúde coletiva: uma análise das teses e dissertações produzidas

(a) O ENS foi constituído atendendo aos dispositivos regimentais da Universidade. O primeiro Conselho foi integrado pelos seguintes membros:

Profa. Dra. Yoriko Kamiyama (Diretora da EEUSP), Profa.

Dra. Maria Jacyra de Campos Nogueira (Chefe), Profa. Dra. Emiko Yoshikawa

Egry (Vice-chefe), Profa. Dra. Hisako Shima (representante da categoria de Professores Assistentes Doutores), Profa.

Rosa Maria Godoy

Serpa da Fonseca (representante da categoria de Professores Assistentes), e docente Elizabeth Fujimori(representante da categoria de auxiliares de ensino).

(b) Integrantes da primeira turma de mestrado: Lúcia Yasuko Izumi Nichiata (auxiliar de ensino do Departamento ENS),

Ana Luiza Aranha e Silva (enfermeira assistencial em saúde mental), Patrícia Aparecida Longa (enfermeira da ARS-8 da Secretaria Municipal de Saúde de S. Paulo). Todas concluíram o programa e duas mestres em saúde coletiva da primeira turma são hoje professoras doutoras pertencentes ao quadro docente da EEUSP.

(c) O valor do crédito à época era de 12 horas.

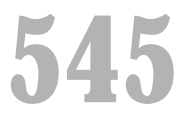

Rev Esc Enferm USP 2005; 39(Esp.):544-52. 
Emiko Yoshikawa Egry Rosa Maria G.S. Fonseca Maria Rita Bertolozzi Maria Amélia de C. Oliveira Renata Ferreira Takahashi
Entre seus docentes, a área de enfermagem em saúde coletiva conta hoje com seus próprios egressos, pois, à medida que foram se qualificando, as docentes do Departamento paulatinamente foram sendo credenciadas para a orientação de mestres e doutores.

Nesse período de 12 anos, muito se produziu e muito se contribuiu para a construção do conhecimento em Saúde Coletiva, nas diferentes linhas de pesquisa que foram se consolidando e ampliando, em decorrência dos estudos que se valeram das categorias de aproximação dos objetos da Saúde Coletiva, como classe social, gênero e geração.

No ano em que se comemora o $30^{\circ}$ aniversário Programa de Pós-graduação em Enfermagem da EEUSP, cabe ponderar: Quais objetos têm sido privilegiados nestes estudos? Quais foram as bases teórico-metodológicas mais férteis em termos de investigação? Quais os campos empíricos que deram voz à realidade? Pesquisou-se para evoluir ou pesquisou-se para superar?

Estas e outras indagações instigaram este estudo, que teve como objetivo analisar a produção do saber da Enfermagem em Saúde Coletiva resultante da pós-graduação do Departamento ENS.

\section{METODOLOGIA}

O estudo foi realizado por meio de revisão bibliográfica, utilizando como referencial filosóficometodológico a hermenêutica-dialética, entendida como um caminho do pensamento e via de encontro entre as ciências sociais e a filosofia que, no presente, busca:

\begin{abstract}
a compreensão e o sentido que vêm do passado, por meio de uma determinada visão de mundo, como possibilidade de propor a transformação valendose de instrumentos de análise dos fenômenos observados. Marx chama atenção para o dinamismo do conhecimento, que só tem sentido como possibilidade de transformação da realidade, portanto, nem somente teoria, nem somente prática, mas união indissolúvel para a fecundi-dade de ambas ${ }^{(4)}$.
\end{abstract}

A hermenêutica consiste na explicação e interpretação de um pensamento e trata da busca dos sentidos existentes na comunicação entre os seres humanos ${ }^{(4)}$.

O fato de pertencermos a um determinado grupo social, a um determinado tempo histórico e de possuirmos determinada formação, faz com que a compreensão hermenêutica seja inevitavelmente condicionada pelo contexto do analista". Assim como Marx, Gadamer chama a atenção para o dinamismo do conhecimento, que só tem sentido como possibilidade de transformação da realidade. A hermenêutica aqui utilizada é a crítica, ou seja, dialética, aquela em que o intérprete busca "entender o texto, a fala, o depoimento como resultado de um processo social (trabalho e dominação) e de um processo de conhecimento (expresso em linguagem), ambos frutos de múltiplas determinações... ${ }^{(4)}$.

As teses e dissertações defendidas no período de 1992 a 2004, produto da área de concentração em Saúde Coletiva e dos doutorados orientados pelas docentes credenciadas junto ao Departamento em Enfermagem em Saúde Coletiva, constituíram a base empírica dos dados. Para inclusão no estudo foram consideradas todas as teses e dissertações originárias da área de influência do Departamento de Enfermagem em Saúde Coletiva e não apenas da área de abrangência. Incluiu-se a produção daqueles alunos que, embora matriculados no mestrado em Saúde Coletiva, em razão do tema, foram orientados por docentes não pertencentes à Área.

As informações foram obtidas dos resumos das teses e dissertações, organizadas em uma ficha de coleta contendo: dados gerais (autora, tipo, data de defesa), dados do resumo (tipo de estudo, objeto de estudo e objetivos, referencial metodológico, técnica de coleta de dados, fonte dos dados, tratamento dos dados, tipo de análise, coerência entre objetivos e resultados e palavras-chave). Os resumos foram obtidos dos volumes originais salvo 3 que foram coletados do banco de dados do sistema Delphos da EEUSP. O período de coleta de dados foi de março a julho de 2004.

\section{DISCUSSÃO DOS RESULTADOS}

\section{A formação de mestres e doutores pelo Departamento de Enfermagem em Saúde Coletiva}

Do início da Área de Concentração Enfermagem em Saúde Coletiva do Programa de Pós-graduação em Enfermagem da EEUSP (PPGE), em 1992 a julho de 2004, foram produzidas 86 teses e dissertações vinculadas à área. As docentes do Departamento ENS foram responsáveis pela formação de 82 alunos, 48 (58,5\%) de Mestrado e 34 (41,5\%) de Doutorado, integrantes tanto da Área de Concentração quanto do Programa Interunidades de Doutoramento em Enfermagem dos campi de São Paulo e Ribeirão Preto da Universidade de São Paulo. Quatro alunas de Mestrado foram orientadas por docentes que não integravam o quadro do Departamento. Como seria de esperar, constata-se que a demanda por Mestrado, primeiro nível da pós-graduação senso estrito, é maior que para o Doutorado. 


\section{Orientação por docente}

No período estudado, a média de orientações de mestrado foi de 3,4 alunos por docente e, de doutorado, 2,4. Ressalte-se que as duas professoras titulares do Departamento concentram um número maior de orientações (33,0\%), sendo responsáveis pela produção de 24 teses de doutorado. Tais professoras também integram o Programa Interunidades de Doutoramento em Enfermagem, no qual vêm pro- duzindo conhecimentos em Saúde Coletiva na perspectiva de gênero e da historicidade dos fenômenos sociais. Quando se verifica a produção das demais docentes, a média de orientações de mestrado é de 3,7 alunos por docente e, de doutorado, $0,8 \%$. Para este conjunto, a média reduzida de orientações de doutorado pode ser explicada, em parte, porque a maioria obteve título de doutor a partir de 1997 (Gráfico 1).

Gráfico 1 - Dissertações de Mestrado e Teses de Doutorado produzidas no Departamento ENS, segundo as docentes orientadoras, Área de Concentração Enfermagem em Saúde Coletiva, 1992 a 2004 - São Paulo, 2004.

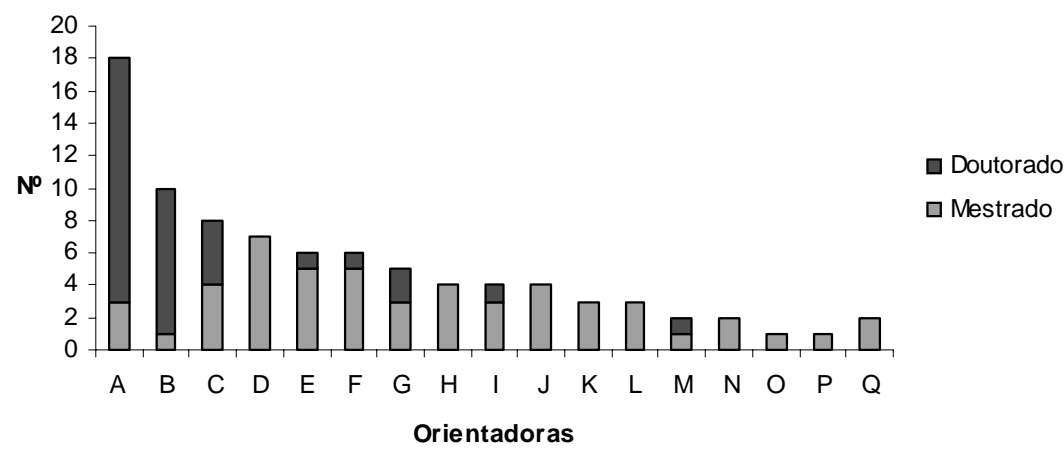

\section{Número de defesas por ano}

O ano de 2002 concentrou aproximadamente um quarto das defesas $(24,4 \%)$, seguido dos anos de 1999 (16,3\%) e 2003 (11,6\%). Houve um evidente incremento da produção a partir de 1999, com 65 produtos, o que corresponde a $75,6 \%$ do total, conforme pode ser verificado no Gráfico 2. Isto se deveu à expansão da Área de Concentração que pas- sou a contar com um número maior de orientadores e oferecer mais disciplinas. O incremento da produção também pode ser atribuído à constituição de grupos de pesquisa no Departamento, como resultado da consolidação das linhas de pesquisa. O processo de consolidação de uma área de concentração no campo da Saúde Coletiva é particularmente complexo, por se tratar de mudança de paradigma, constituindo um campo novo de conhecimentos ${ }^{(6)}$.

Gráfico 2 - Dissertações de Mestrado e Teses de Doutorado do Departamento ENS segundo o ano de defesa, Área de Concentração Enfermagem em Saúde Coletiva, 1992 a 2004 - São Paulo, 2004.

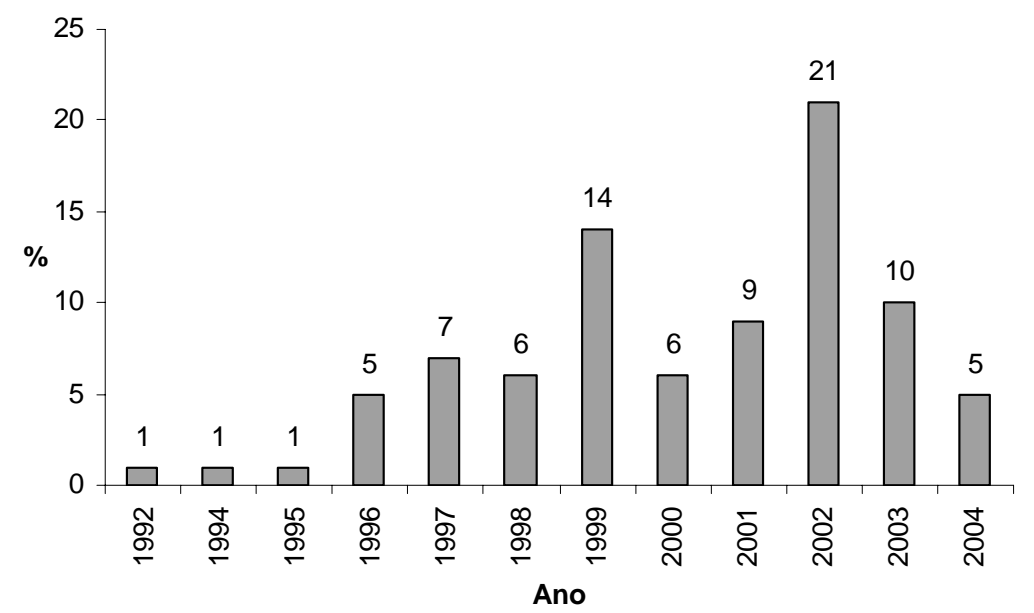

Construindo o conhecimento em saúde coletiva: uma análise das teses e dissertações produzidas 
Emiko Yoshikawa Egry Rosa Maria G.S. Fonseca Maria Rita Bertolozzi Maria Amélia de C. Oliveira Renata Ferreira Takahashi

\section{Objetos de Estudo}

As pesquisas que originaram teses e dissertações ocuparam-se de uma ampla variedade de objetos de investigação. Dentre os 96 objetos de estudo identificados, foram mais freqüentes os relativos aos perfis epidemiológicos e de reprodução social (32,3\%), que englobam estudos sobre perfis de saú- de-doença, reprodução social, condições de vida, trabalho e saúde, as inter-relações da determinação social da saúde-doença na explicação das manifestações clínicas de determinadas doenças, além de estudos sobre as carências alimentares ou padrões alimentares inadequados, em função da inserção social (Tabela 1).

Tabela 1 - Objetos estudados nas teses e dissertações, Área de Concentração Enfermagem em Saúde Coletiva, 1992 a 2004 - São Paulo, 2004.

\begin{tabular}{lcc}
\hline \multicolumn{1}{c}{ Objetos estudados } & N & \% \\
\hline Perfis epidemiológicos e reprodução social & 31 & 32,2 \\
Trabalho e saúde & 14 & 14,5 \\
Educação: qualificação, capacitação e ensino & 11 & 11,5 \\
Aids: informação e vulnerabilidade & 9 & 9,4 \\
Gerência, modelos e organização da atenção & 7 & 7,3 \\
Saberes, competências, ética e ideologias & 6 & 6,3 \\
Saúde da Mulher & 5 & 5,2 \\
Saúde da criança, escolar e adolescente & 4 & 4,2 \\
Necessidade em saúde & 3 & 3,1 \\
Infecções e isolamento & 3 & 3,1 \\
Outros & 3 & 3,1 \\
\hline Total & $\mathbf{9 6}$ & $\mathbf{1 0 0 , 0 0}$ \\
\hline
\end{tabular}

Em segundo lugar, estão os estudos que se ocupam do trabalho em saúde e da relação do trabalho com a saúde (14,5\%). As questões relativas à educação em geral dos trabalhadores da saúde e os processos de qualificação e capacitação encontramse em terceiro lugar, com $11,5 \%$. O tema aids foi tomado como objeto de investigação por meio de sistemas de informação, vulnerabilidades, transmissão vertical e representação social e ocupa um lugar de destaque em 9,4\% das teses e dissertações.

Identifica-se uma preocupação dos autores com a gerência, os modelos e organização da atenção nos Programas de Saúde da Família, no Núcleo de Epidemiologia e Informação (NEPI), assim como questões relativas ao sistema de referência e contra-referência (7,3\%). Em menor número, ainda que importantes na construção do campo da Saúde Coletiva, encontram-se estudos relativos aos saberes, competências, ética e ideologias em Saúde Coletiva (6,3\%), além de outros que buscaram decodificar o conceito-horizonte de necessidades em saúde.

A pluralidade de ângulos a que devem se submeter esses objetos requer instrumentais analíticos que integram saberes e métodos das disciplinas científicas que se entrecruzam na Saúde Coletiva, principalmente das Ciências Sociais, identificados nos estudos analisados.

Por outro lado, a expressão que apresentam os estudos epidemiológicos tem sentido porque a
Epidemiologia é uma das áreas nucleares da Enfermagem em Saúde Coletiva. Não se tratando de mera utilização asséptica do instrumental da Epidemiologia, constitui-se de um movimento de adesão à necessidade de produção do conhecimento a partir de uma perspectiva inovadora, iniciada na década de 70 do século passado, por ocasião da constituição da Epidemiologia Crítica ou Social.

A preocupação com a superação das desigualdades sociais impulsionou o desenvolvimento de estudos cujos objetos emergem das contradições sociais e apontam para intervenções, conforme o projeto da Saúde Coletiva. Evidenciam-se, portanto, análises macro-sociais (estruturais), que buscam as determinações dos fenômenos sociais. A própria constituição do saber da Enfermagem em Saúde Coletiva, que foi impulsionada pelo grupo de docentes da Escola de Enfermagem da USP, mostra esse tipo de produção.

Ao longo dos anos, verifica-se maior articulação entre os processos nas três dimensões da realidade $^{(5)}$ - estrutural, particular e singular - com a preocupação constante de não estabelecer relações mecanicistas entre essas dimensões, ao contrário, considerando as mediações entre planos individual, dos grupos sociais, institucionais, políticos, simbólicos e ideológicos. Além disso, o compromisso com o projeto da Saúde Coletiva, das autoras, é evidente, uma vez que na maior parte dos estudos, os indivíduos foram considerados como sujeitos sociais. 
A apreensão da realidade de saúde, assim como das condições de vida e trabalho da população, subsidiam o planejamento, a proposição e a intervenção em saúde de acordo com os princípios e diretrizes do Sistema Único de Saúde. Os estudos também inovam ao construir indicadores mais adequados à compreensão da realidade, ainda que esse campo careça de aprofundamento, principalmente no que diz respeito às abordagens que dêem conta da dimensão social e política, articuladas às questões que emanam das necessidades e problemas de saúde dos sujeitos sociais no cotidiano. Revela-se, em algumas pesquisas, a abordagem que transcende 0 espaço institucional e que busca, no território, as necessidades de saúde da população.

É interessante verificar que a produção departamental segue o padrão de produção do conhecimento próprio da Saúde Coletiva. Pellegrini Filho e colaboradores ${ }^{(7)}$, em meados da década de 1980, ao avaliar a pesquisa no campo da Saúde Coletiva, identificaram predominância de estudos sobre condições materiais de vida e de trabalho e sua associação com a saúde-doença.

Gráfico 3 - Dissertações de Mestrado e Teses de Doutorado do Departamento ENS segundo o referencial teórico-metodológico adotado, Área de Concentração Enfermagem em Saúde Coletiva, 1992 a 2004 - São Paulo, 2004.
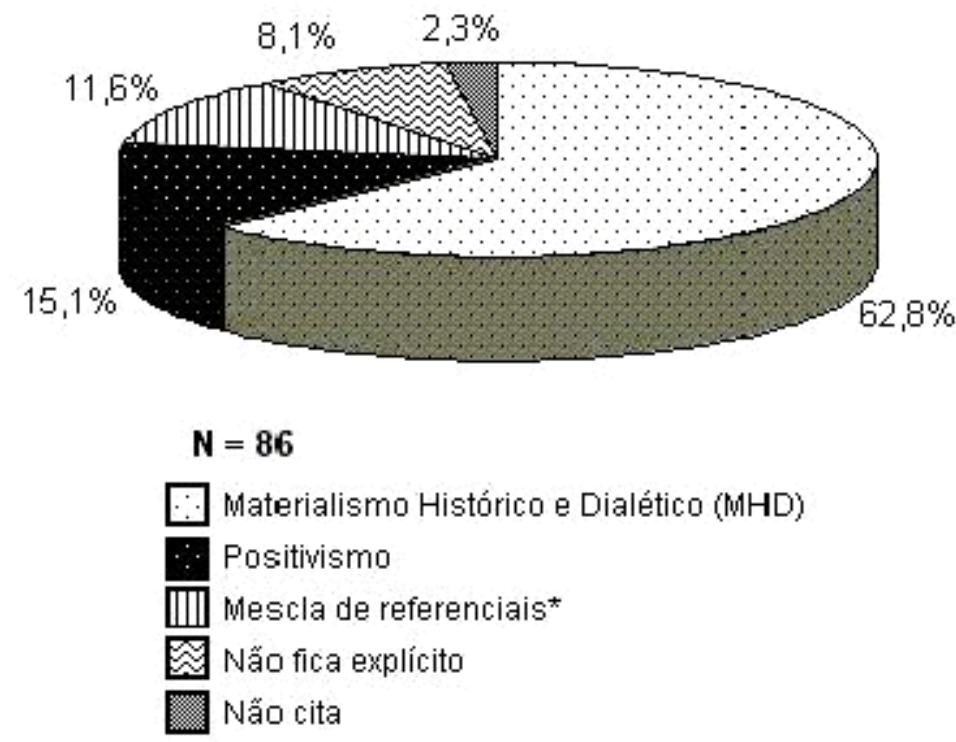

Se a perspectiva departamental foi a construção desse novo campo de saberes e práticas - a Saúde Coletiva - embasada no materialismo histórico e dialético, houve significativo avanço em termos das concepções filosóficas. No primeiro estudo departamental realizado entre 1989 a 1992, lastreada na pesquisa participante e tendo por objeto a integração docente-assistencial (IDA) como referencial
Constituindo uma outra marca da produção do Departamento, diferentes objetos de estudo são abordados na perspectiva de gênero, sejam os relativos aos fenômenos específicos da vida das mulheres (aborto, reprodução biológica, violência, mortalidade materna), sejam aqueles acerca do trabalho da Enfermagem como profissão feminina (condições de vida e trabalho das enfermeiras).

\section{Referencial teórico-metodológico e tipos de estudos}

O referencial teórico-metodológico mais adotado foi o materialismo histórico e dialético (54,63\%), seguido do positivismo $(13,15 \%)$ e da mescla dos referenciais $(10,12 \%)$. Neste conjunto foram abrigados os estudos cujo referencial tomado era simultaneamente dois dos componentes: MHD, legislação, determinantes da epidemia, perspectiva construtivista-histórica, promoção da saúde; teoria críticosocial dos conteúdos, gênero, teoria das representações sociais, vulnerabilidade (Gráfico 3).
Construindo o conhecimento em saúde coletiva: uma análise das teses e dissertações produzidas teórico-metodológico do ensino de enfermagem, as pesquisadoras já haviam concluído que:

as diversas formas de representar e operacionalizar a dimensão pedagógica da IDA, estão ancoradas em diferentes concepções filosóficas que se traduzem em visões de mundo diferentes. Assim sendo, é possível identificar num dos conjuntos uma forte aproximação com a

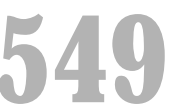

Rev Esc Enferm USP 2005; 39(Esp.):544-52. 
Emiko Yoshikawa Egry Rosa Maria G.S. Fonseca Maria Rita Bertolozzi Maria Amélia de C. Oliveira Renata Ferreira Takahashi visão idealista de mundo e noutro, uma forte aproximação com a visão materialista histórica e dialética. Intermediando-as há um conjunto que mescla categorias de ambas as visões. Na realidade isto é um reflexo das diferentes visões de mundo que permeiam as práticas sociais da sociedade brasileira contemporânea da qual fazem parte os pesquisadores deste estudo (...) A discussão dos três conjuntos conceituais levou os participantes a repensar as contradições existentes no interior do seu discurso e da sua prática de IDA. Assim, (diante dessas três concepções filosóficas) os pressupostos que amarram a visão idealista de mundo não contemplam a reflexão acerca da reorientação da prática da IDA (...) e foram negadas pelo grupo enquanto horizonte de aprofundamento. (...) a visão materialista histórica e dialética foi eleita por uma parcela dos pesquisadores/ pesquisados como horizonte a ser alcançado e por outra parcela, como objeto de aprofundamento no exercício da IDA ${ }^{(8)}$.

Olhar os estudos a partir dessa tipificação permitiu observar, como mostra o Gráfico 4, a predominância de estudos denominados qualitativos, seguidos pelos quantitativos. Se ao primeiro conjunto for somado o que mesclou as duas, $72,1 \%$ dos estudos recorreu de alguma forma aos métodos e bases qualitativas.

Gráfico 4 - Dissertações de Mestrado e Teses de Doutorado do Departamento ENS segundo os tipos de estudos realizados, Área de Concentração Enfermagem em Saúde Coletiva, 1992 a 2004 São Paulo, 2004.
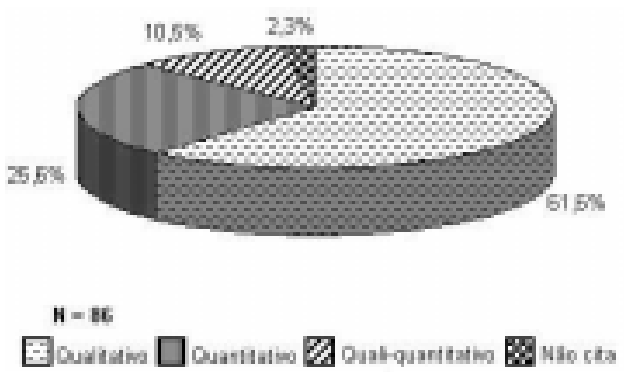

\section{População de estudo, fontes e estratégias de coleta de dados}

Boa parte dos estudos foi desenvolvida em cenários, onde o Departamento de Enfermagem em Saúde Coletiva historicamente desenvolve projetos de Integração Docente-Assistencial no Município de São Paulo. Pode-se verificar, portanto, a devida articulação entre ensino, pesquisa e extensão de serviços à comunidade, levando a cabo a função social da Universidade e correspondendo à expectativa de formação profissional baseado na realidade de saúde e perfis epidemiológicos da população.

A população freqüentemente estudada foi constituída pelos trabalhadores de enfermagem (25,6\%), principalmente enfermeiras, seguidas de auxiliares de enfermagem e da equipe de enfermagem como um todo. Em segundo lugar, os usuários de serviços de saúde $(17,4 \%)$, seguidos de grupos populacionais do território (15,1\%), com destaque para mulheres e crianças. Documentos tais como fichas de notificação compulsória, prontuários clínicos e publicações científicas também foram fontes de dados em $15,1 \%$ dos estudos. Docentes e estudantes do ensino superior constituíram outros grupos estudados em 11,6\% das pesquisas (Gráfico 5).

Gráfico 5 - Dissertações de Mestrado e teses de Doutorado do Departamento ENS segundo o tipo de fonte de dados, Área de Concentração Enferma-gem em Saúde Coletiva, 1992 a 2004 - São Paulo, 2004.
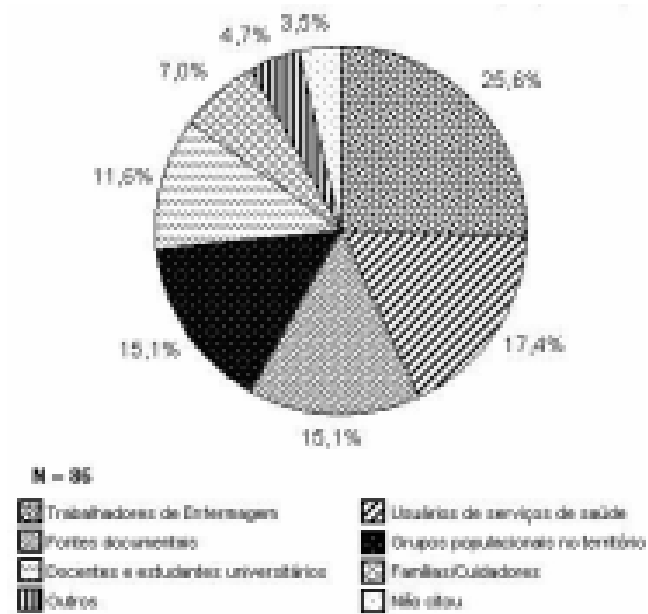

As entrevistas constituíram a principal estratégia para obtenção de dados (54,7\%), embora cerca de $19,8 \%$ dos resumos não tenham citado a estratégia de coleta de dados. Cabe destacar que o Departamento também tem inovado ao utilizar oficinas de trabalho e dinâmicas de grupo para coleta de dados, presentes em 14,0\% dos estudos. A pesquisa documental e a utilização de dados secundários provenientes de bancos de dados foram estratégias de coleta em 9,3\% das pesquisas (Gráfico 6).

Gráfico 6 - Dissertações de Mestrado e Teses de Doutorado do Departamento ENS segundo estratégias para a coleta de dados, Área de Concentração Enfermagem em Saúde Coletiva, 1992 a 2004 - São Paulo, 2004.
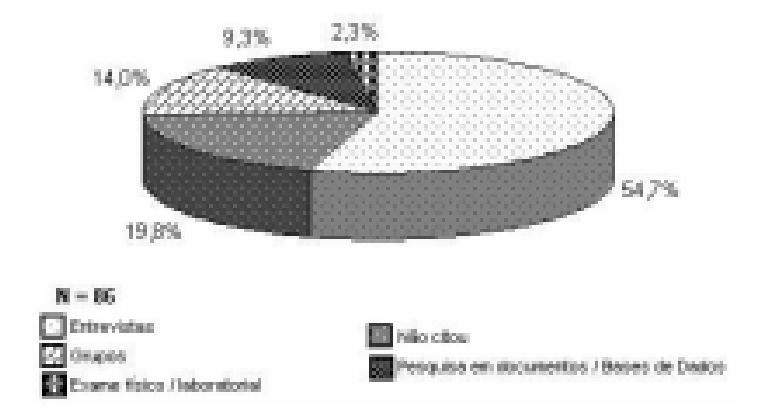

Rev Esc Enferm USP 2005; 39(Esp.):544-52.

D


A maior parcela dos resumos (43,0\%) não menciona a forma de tratamento dos dados. Aproximadamente 27,9\% dos estudos valeram-se de análises qualitativas, principalmente a análise de discurso (15,2\%), seguida da análise de conteúdo (8,1\%). Em 3,5\% dos resumos, a hermenêutica-crítica foi mencionada como método de análise e interpretação dos dados. A análise de freqüência simples foi utilizada em $24,4 \%$ dos casos, em estudos quantitativos. O uso da taxonomia e classificadores foi mencionado nos estudos sobre necessidades em saúde e classificação das práticas de Enfermagem em Saúde Coletiva-CIPESC.

Gráfico 7 - Dissertações de Mestrado e Teses de Doutorado do Departamento ENS segundo as formas de tratamento dos dados utilizadas, Área de Concentração Enfermagem em Saúde Coletiva, 1992 a 2004 - São Paulo, 2004.

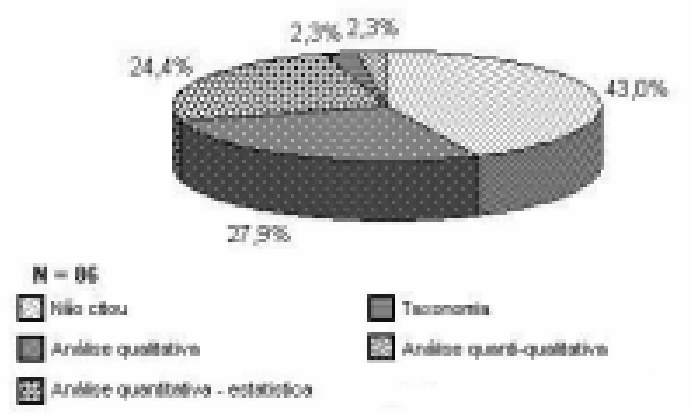

\section{CONCLUSÕESE CONSIDERAÇÕES FINAIS}

A grande maioria das teses e dissertações da Área utilizou as categorias relativas à produção e reprodução social, assim como as demais categorias férteis como gênero e geração, representado um avanço teórico importante, pois possibilitam a aproximação do objeto fenomênico à luz dos pressupostos do materialismo histórico e dialético. Pode-se dizer que algumas avançaram mais ainda, no sentido de renovar a episteme da saúde coletiva, incorporando instrumentais que não se reduzem à interpretação, mas assumem uma dimensão histórica de intervenção para a transformação dos perfis de saúde-doença da população e dos processos de trabalho em saúde e educação.

A presença marcante de estudos epidemiológicos evidencia uma trajetória constituinte do campo da Saúde Coletiva. Embora possam ser identificados estudos que interpretam objetos sob o ângulo multicausal, tomando as causas como fatores que marcam diferenças e produzem efeitos, verifica-se a tendência de superação dos reducionismos e dos determinismos, fracionadores e imobilizadores da rea- lidade, ao reificar as "causas" explicativas dos fenômenos.

Por ocasião da emergência do movimento da Saúde Coletiva, muitos estudos demarcavam fortemente o espaço da determinação macroeconômica, muito provavelmente na tentativa de estabelecer um marco teórico sólido, enraizado no marxismo. Breilh( ${ }^{(9)}$ considera que essa politicidade "externa”, coloca os conteúdos dos trabalhos científicos na sociedade e no poder, ou melhor, na relação entre o geral e o particular. A politicidade "interna" aos trabalhos científicos encontra-se na construção do modo de pensar científico e expressa-se na configuração das categorias e explicações que a ciência incorpora. A separação entre a primeira forma de politicidade e a segunda leva ao descuido da congruência interna do trabalho científico. É contra essa possibilidade que a ciência deve se insurgir, mantendo seu potencial transformador, indo ao encontro das necessidades dos grupos sociais, especialmente os mais vulneráveis. Para o autor, a seleção dos objetos de estudo e as maneiras de construí-los cientificamente estão relacionadas dialeticamente e essa práxis determina a própria construção interna das categorias de análise ${ }^{(9)}$.

Pode-se concluir que se pesquisou mais no sentido de superar ${ }^{(10)}$ e não apenas para evoluir, pois buscou-se: construir uma cultura de transformação desalienada, favorecendo o compromisso com os subalternos; compreender a relação dialética entre o biológico e o social, entre eventos individuais e coletivos; revisar o método científico, produzindo mudança metodológica no percurso, relacionando dialeticamente a quantidade e a qualidade e o teórico e o prático e, realizar a interpretação objetiva da realidade, desvelando o caráter ideológico das práticas e políticas.

Finalmente, ao verificar os avanços proporcionados pelas pesquisas de pós-graduação no Departamento de Enfermagem em Saúde Coletiva, cabe instigar a hermenêutica-crítica, na busca permanente de contradições dos processos de produção dos conhecimentos, pois:

querer captar uma realidade efêmera com conceitos e teorias duradouras ou acabadas é destruir de partida o pensamento através do próprio pensamento. A dialética processual da realidade exigirá, assim, um corpo também processual de categorias, o que quer dizer que toda teoria e metodologia de sua armação não podem ser estatutos fixos, indiscutíveis, normativos absolutamente, mas precariamente válido como é válida uma realidade social(11).
Construindo o conhecimento em saúde coletiva: uma análise das teses e dissertações produzidas 
Emiko Yoshikawa Egry Rosa Maria G.S. Fonseca Maria Rita Bertolozzi Maria Amélia de C. Oliveira Renata Ferreira Takahashi

\section{REFERÊNCIAS}

(1) Escola de Enfermagem da Universidade de São Paulo. Departamento de Enfermagem em Saúde Coletiva. Ata da sessão de instalação e $1^{\mathrm{a}}$. reunião ordinária do Conselho do Departamento de Enfermagem em Saúde Coletiva da Escola de Enfermagem da Universidade de São Paulo, realizada aos treze dias de fevereiro de mil novecentos e oitenta e sete. São Paulo; EEUSP/ ENS; 1987.

(2) Egry EY. A pós-graduação em enfermagem em saúde coletiva: o desafio da construção conjunta do conhecimento. Rev Esc Enferm USP 1996; 30 (n. esp): 59-62.

(3) Miyadahira AMK, Silva MJP, Egry EY. A pós-graduação stricto sensu em Enfermagem comemora 30 anos! [Editorial] Rev Esc Enferm USP 2004; 38(10):7.

(4) Minayo MCS. O desafio do conhecimento: pesquisa qualitativa em saúde. $4^{\mathrm{a}}$ ed. São Paulo: Hucitec; 1996.

(5) Egry EY. Saúde coletiva: construindo um novo método em Enfermagem. São Paulo: Ícone; 1996.

(6) Pellegrini Filho AAlmeida C, Szklo F, Viacava F, Cordeiro $H$, Nicoletti $L$ et al. Pesquisa em saúde coletiva. In: Associação Brasileira de Pós-Graduação em Saúde Coletiva. Estudos de saúde coletiva. Rio de Janeiro: Abrasco; 1986a. v. 4. p. 41-50.
(7) Pellegrini Filho A, Pacheco M, Szklo F, Buss PM, Nunes ED. Reunião de avaliação do Programa de Saúde Coletiva FINEP/CNPq: relatório final. In: Associação Brasileira de Pós-Graduação em Saúde Coletiva. Estudos de saúde coletiva. Rio de Janeiro: Abrasco; 1986b. v. 4. p. $70-89$.

(8) Egry EY, Shima H, organizador. Integração docenteassistencial como referencial teórico-metodológico para o ensino e a pesquisa da assistência de enfermagem em saúde coletiva. In: Universidade de São Paulo. Escola de Enfermagem. Integração docenteassistencial. São Paulo: Escola de Enfermagem da USP; 1992. p. 77-131.

(9) Breilh J. La epidemiología critica latino-americana. In: Franco S, Breilh J, Laurell AC, editores. Debates en medicina social. Quito: OPAS, 1991. p. 164-215.

(10) Egry EY. Pesquisar para evoluir? Pesquisar para superar! Rev Esc Enferm USP 1992: 26 (n. esp):14151.

(11) Demo P. Metodologia científica em ciências sociais. São Paulo: Atlas; 1985.
Correspondência: Emiko Yoshikawa Egry Av. Dr. Enéas de C. Aguiar, 419 - Cerqueira César - São Paulo 05403-000 - SP

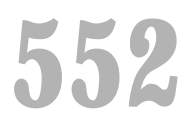

Rev Esc Enferm USP 2005; 39(Esp.):544-52. 